\title{
Divergent Asymmetric Synthesis of Polycyclic Compounds via Vinyl Triazenes
}

\author{
David Kossler, ${ }^{+[a]}$ Florian G. Perrin, ${ }^{+[a]}$ Abdusalom A. Suleymanov, ${ }^{[a]}$ Gregor Kiefer, ${ }^{[a]}$ Rosario \\ Scopelliti, ${ }^{[a]}$ Kay Severin ${ }^{\star[a]}$ and Nicolai Cramer*[a]
}

\begin{abstract}
Vinyl triazenes are obtained by enantioselective [2+2] cycloaddition reactions of bicyclic alkenes with 1-alkynyltriazenes in the presence of a Ru"-catalyst with a chiral cyclopentadienyl ligand. The triazenes serve as unique vinyl cation surrogates. Under acidic conditions, the triazene functionality can be replaced by a variety of groups including halides, alkoxides, sulfoxides, amides, arenes and heteroarenes, providing efficient access to a pool of chiral polycyclic compounds.
\end{abstract}

Aromatic compounds with 3,3-dialkyltriazene groups are valuable reagents in synthetic organic chemistry. ${ }^{[1]}$ Triazenes are base-resistant and compatible with organometallic reagents and catalysts. ${ }^{[2]}$ It is thus possible to functionalize the arene ring under a variety of conditions. In the presence of acid, however, the triazene group is cleaved to give intermediate diazonium salts, which can be elaborated to substituted arenes (Scheme 1). ${ }^{[1]}$ Numerous functional groups can be introduced in that fashion, making aromatic triazenes interesting and valuable intermediates for the late-stage diversification of arenes. In analogy, vinyl triazenes are expected to display a similar reactivity pattern. Protonation of the triazene followed by cleavage of the nitrogen-nitrogen bond would give unstable vinyl diazonium compounds, ${ }^{[3]}$ which could be intercepted with different nucleophiles. In fact, vinyl triazenes were used successfully as masked vinyl cations, ${ }^{[4]}$ but compared to the well-established chemistry of 1-aryltriazenes, there are only very scarce reports about the synthesis and reactivity of vinyl triazenes. ${ }^{[4-6]}$ Notably, the utilization of alkenyl triazenes as intermediates for divergent organic syntheses is completely unexplored. We have recently introduced a method allowing the preparation of 1-alkynyltriazenes from lithium amides, nitrous oxide, and alkynyl Grignard reagents. ${ }^{[5]}$ Subsequently, we have shown that 1-alkynyltriazenes are activated alkynes with a reactivity profile similar to ynamides. ${ }^{[6]}$ We were intrigued by the prospect of preparing chiral vinyl triazenes from 1-alkynyltriazenes using catalytic enantioselective transformations. If the arising alkenyl triazenes would behave as

[a] D. Kossler, F. G. Perrin, A. A. Suleymanov, Dr. G. Kiefer, Dr. R. Scopelliti, Prof. Dr. K. Severin, Prof. Dr. N. Cramer Institut of Chemical Sciences and Engineering (ISIC) École Polytechnique Fédérale de Lausanne (EPFL) $\mathrm{CH}-1015$ Lausanne (Switzerland) E-mail: kay.severin@epfl.ch

Homepage: http://lcs.epfl.ch/home

E-mail: nicolai.cramer@epfl.ch Homepage: http://isic.epfl.ch/lcsa

[+] These authors contributed equally to this work.

Supporting information for this article is given via a link at the end of the document. vinyl cation surrogates, they would be highly valuable intermediates for the divergent synthesis of chiral compounds.

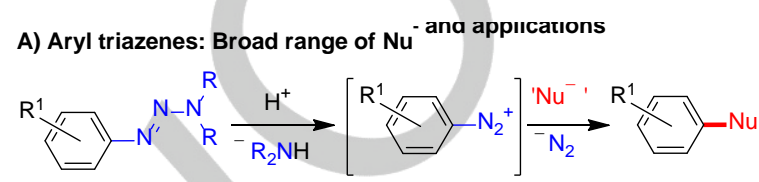

B) Alkenyl triazenes: Largely underdeveloped

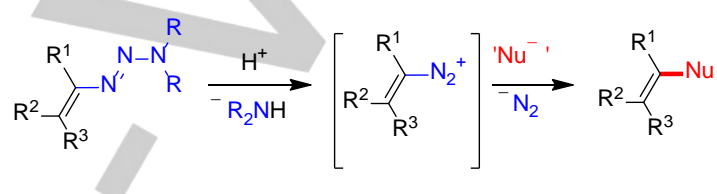

Scheme 1. Reactivity of aromatic triazenes (A) and alkenyl triazenes (B).

Starting point of our investigations was the recent discovery that [2+2] cycloaddition reactions between strained olefins and certain alkynes can be performed with high enantioselectivity by using a ruthenium catalyst with a chiral cyclopentadienyl ligand. ${ }^{[7,8]}$ The compatibility and behavior of 1-alkynyltriazenes with transition metal-catalyzed reactions had not been studied before. Therefore, we first examined the reaction of 1,4-dihydro1,4-epoxynaphthalene (1a) with 3,3-diisopropyl-1-(phenylethynyl)triazene (2a) in the presence of the achiral complex $\mathrm{Cp}^{*} \mathrm{Ru}(\mathrm{COD}) \mathrm{Cl}$ (Equation 1). This $\mathrm{Ru}(\mathrm{II})$ complex is the common catalyst of choice for [2+2] cycloaddition reactions. ${ }^{[9,10]}$ Complete conversion was detected after one hour at ambient temperature, forming $75 \%$ yield of cyclobutene 3aa.

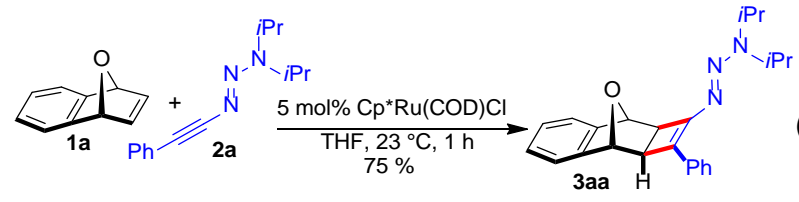

The good compatibility of 1-alkynyltriazenes with Ru(II)catalyzed cycloaddition reactions prompted us to investigate enantioselective transformations with chiral cyclopentadienyl ruthenium complexes. Employing phenyl-substituted $\mathrm{Cp}^{\mathrm{x}}$ ligand bearing catalyst $\mathbf{R u}(5 \mathrm{~mol} \%)$, we investigated the influence of the counterion (Table 1, for a full ligand screen and optimization, see $\mathrm{SI}$ ). The cationic parent catalyst delivered vinyl triazene 3aa with high enantioselectivity of 94:6 er, but the yield was poor $(17 \%)$. As observed previously, ${ }^{[7 a]}$ additives such as $\left(\mathrm{Bu}_{4} \mathrm{~N}\right) \mathrm{Cl}$ and $\left(\mathrm{Bu}_{4} \mathrm{~N}\right) \mathrm{Br}$ rendered the catalyst dramatically more active, providing full conversion in less than $30 \mathrm{~min}$ at $0^{\circ} \mathrm{C}$. Most likely the catalytic activity of the $\mathrm{Ru}$ complex is enhanced by coordination of the halide anions to the metal center. 
Table 1: Optimization of the enantioselective vinyl triazene formation. ${ }^{[a]}$

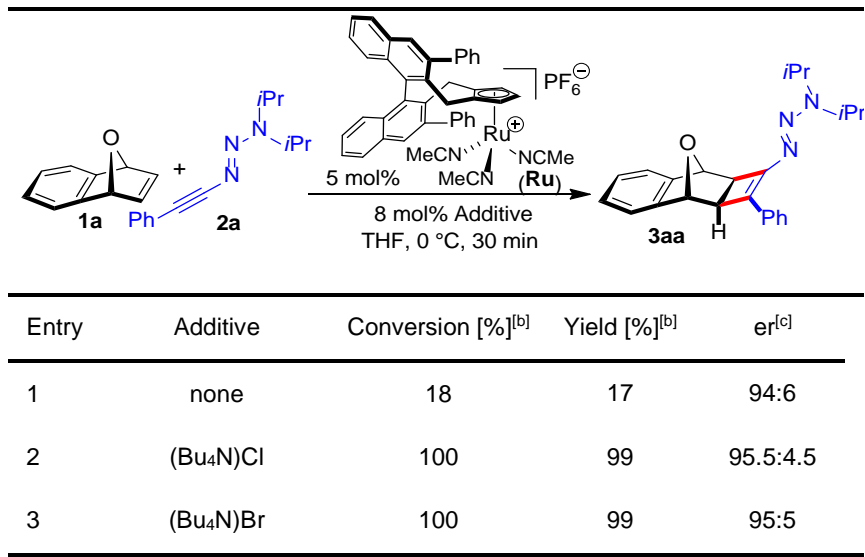

[a] Conditions: $30 \mu \mathrm{mol} \mathbf{1 a}, 25 \mu \mathrm{mol} \mathbf{2 a}, 2.0 \mu \mathrm{mol}$ additive, $1.25 \mu \mathrm{mol} \mathbf{R u}$ $0.3 \mathrm{M}$ in THF, $0{ }^{\circ} \mathrm{C}, 30 \mathrm{~min}$; [b] Determined by ${ }^{1} \mathrm{H}-\mathrm{NMR}$ with an internal standard; [c] Determined by chiral HPLC.

The scope of the enantioselective cyclobutene formation was investigated next (Scheme 2). Replacing the iPr groups of the alkynyl triazene $\mathbf{2 a}$ by larger cyclohexyl groups (2b) slightly increased the selectivity to $96: 4 \mathrm{er}$. However, the reactivity of this bulkier triazene was a bit lower. The smaller Me/iPrsubstituted triazene 2c caused a drop in enantioselectivity. Moreover, the stability of product $3 a c$ is decreased, reflected by a lower yield of $48 \%$. The aromatic alkynyl substituent $\mathrm{R}^{1}$ can be varied from an electronically modulated arene (3ad and 3ae) to a heteroarene (3af). Alkyl substituents alkynes (2g and $\mathbf{2 h}$ ) are less reactive and require ambient reaction temperature. The selectivity of 99:1 er for the cyclohexyl-substituted product $\mathbf{3 a g}$ is excellent. The bicyclic olefins $1 x$ can be varied as well. For instance, dibrominated cyclobutene $3 \mathbf{c a}$ was obtained with 97.5:2.5 er. X-Ray crystallographic analysis of 3 ca allowed the unambiguous determination of its absolute configuration. ${ }^{[11]}$ Diester-substituted alkene $\mathbf{3 b}$ gave cyclobutene $\mathbf{3 b a}$ in $84 \%$ yield and 94.5:5.5 er. In this case, the addition of a catalytic amount of $\mathrm{MgBr}_{2}$ dramatically enhanced the reactivity, suggesting a coordination of the Lewis acid to the substrate. Additionally, norbornene $\mathbf{1 d}$ was a suitable substrate. To compare the reactivity and selectivity of alkynyltriazenes with established nitrogen-substituted alkynes, ynamide $2 \mathbf{i}$ was subjected to the same conditions. Cyclobutene 3ai was obtained in good yield, however with a largely inferior enantiomeric ratio of $77.5: 22.5$.
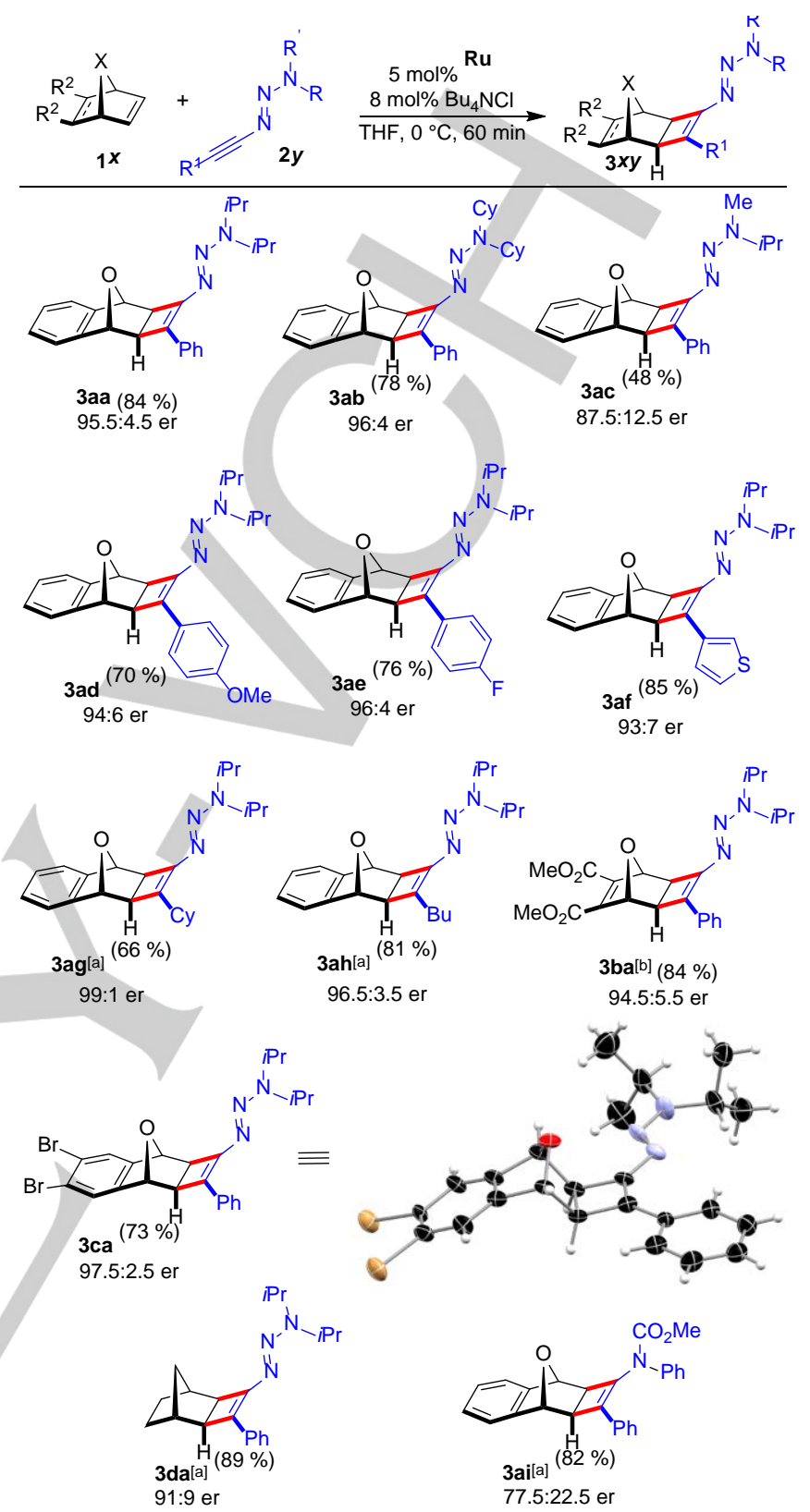

Scheme 2. Scope of the enantioselective [2+2] cyclization: $0.1 \mathrm{mmol} 1 \boldsymbol{x}, 0.12$ $\mathrm{mmol}$ of $2 y, 5.00 \mu \mathrm{mol} \mathrm{Ru}, 8.00 \mu \mathrm{mol} \mathrm{Bu} \mathrm{NCl}, 0.3 \mathrm{M}$ in THF, $0{ }^{\circ} \mathrm{C}$ for $60 \mathrm{~min}$ Isolated yield; er determined by chiral HPLC; [a] at $23^{\circ} \mathrm{C}$; [b] $15.0 \mu \mathrm{mol} \mathrm{MgBr}_{2}$.

The catalyst loading for the enantioselective vinyl triazene formation could be lowered to $0.75 \mathrm{~mol} \%$ without any detrimental effect on the selectivity (Equation 2). The high crystallinity of the triazene products enables upgrade of the enantiomeric enrichment to >99.5:05 er. 


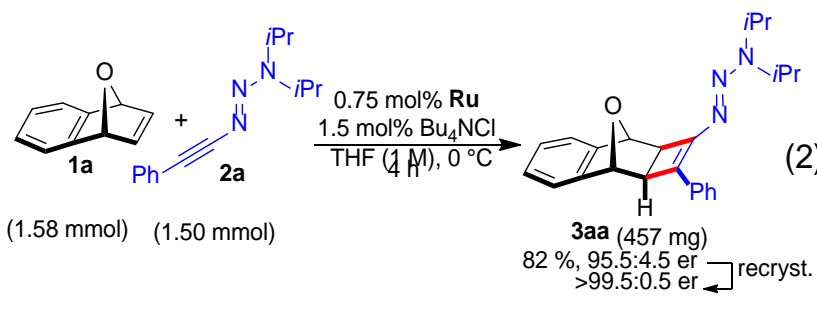

The convenient access to chiral vinyl triazenes $\mathbf{3}$ allowed targeting their divergent elaboration. Protonation of $\mathbf{3}$ should lead to the corresponding vinyl diazonium salt, which would give a highly reactive vinyl cation upon release of dinitrogen. We investigated a broad range of nucleophiles for their suitability to intercept the vinyl cation intermediate (Scheme 3). For instance, exposure of 3aa to hydrochloric acid provided vinyl chloride 4 . Analogously, vinyl iodide $\mathbf{5}$ and bromide $\mathbf{6}$ were obtained by reaction with $\mathrm{HI}$ and $\mathrm{HBr}$, respectively. Alkenyl fluoride 7 was obtained by addition of HF-pyridine. The vinyl halides are likewise valuable platform intermediates. For example, vinyl bromide 6 reacted smoothly in a follow-up Suzuki reaction, giving cross-coupled product $\mathbf{8}$. The direct introduction of carbon nucleophiles was achieved via Friedel-Crafts-type reactions by protonation of the triazene with TFA in presence of suitable arenes. For instance, protonation of $\mathbf{3 a h}$ in mesitylene gave rise to 9 , and coupling reactions with thiophene lead to $10 \mathrm{a}$ and $10 \mathrm{~b}$ The introduction of oxygen and sulfur nucleophiles was achieved by protonation of the triazene with TFA. The interception was demonstrated with methanol and pentanethiol delivering enol ether 13 and vinyl sulfide 11. With water as nucleophile, cyclobutanone 14 was obtained as a single diastereomer. ${ }^{[11]}$ Performing the protonation in wet acetonitrile induced a clean Ritter-type reaction delivering amides $15 \mathrm{a}$ and $\mathbf{1 5 b}$. The reactions outlined in Scheme 3 focus on the triazenes 3aa and 3ah. Further investigations with the racemic version of the norbornene-derived triazene 3da show that similar substitution reactions can be achieved with other [2+2] cycloaddition products (for details see SI).

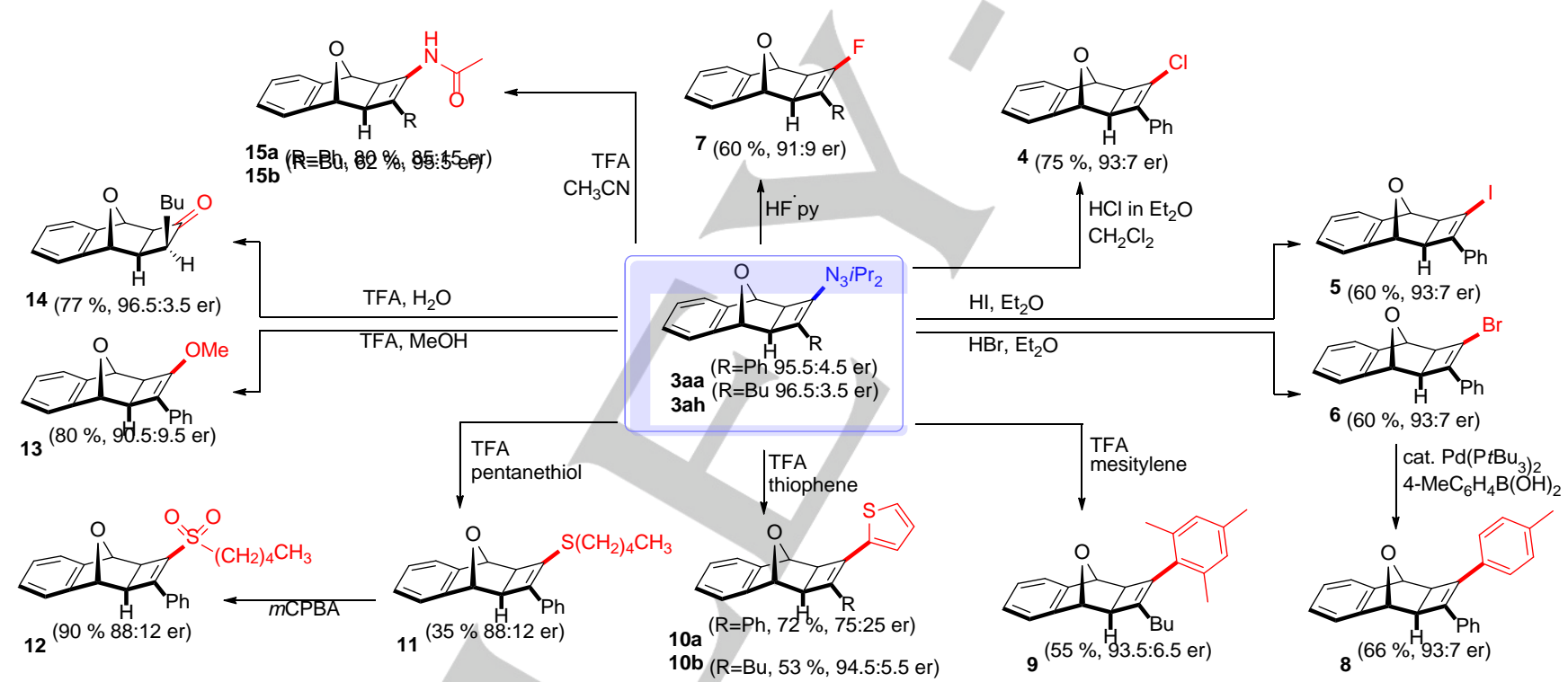

Scheme 3. Flexible conversion of vinyl triazenes into a variety of target structures.

In most cases, good to excellent enantiospecificity was observed for these substitution reactions. However, depending on the nucleophilicity of the trapping reactant and on the substituent of the cyclobutene, some erosion of the enantioselectivity was detected. It was most visible with particularly weak nucleophiles and for triazenes featuring a phenyl substituent adjacent to the triazene function. These observations provide compelling evidence for the proposed vinyl cation intermediate. Protonation and dissociation of the secondary amine produces alkenyl diazonium salt 16 (Scheme 4). Subsequently, $\mathrm{N}_{2}$ and cyclobutenyl cation $\mathbf{1 7}$ are formed. Racemization can occur by two pathways. Either a 1,2-shift with a migrating group R (18) or reversible formation of the cyclopropylidenemethyl cation 19 would lead to ent-17. ${ }^{[12]}$ In the presence of a good nucleophile and/or in the absence of a stabilizing phenyl group $(R=P h)$, the trapping reaction is significantly faster than the racemization, allowing the reaction to proceed with high enantiospecificity.

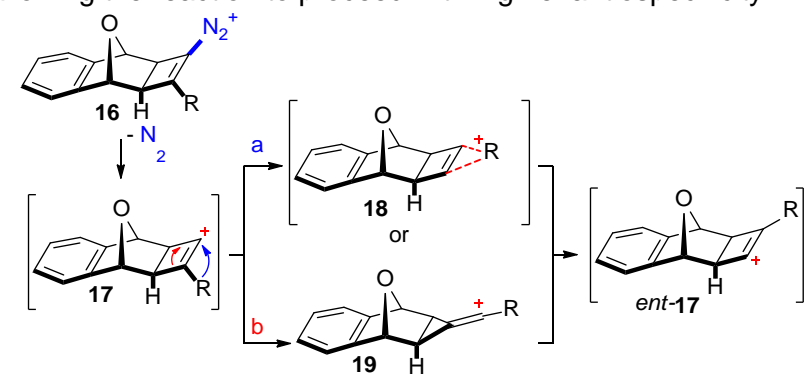

Scheme 4. Possible racemization mechanism of the vinyl cation intermediate 17 
The enantioselective cyclization and triazene hydrolysis can be performed as a one-pot transformation (Scheme 5). For instance, cyclobutanone 14 was obtained in $67 \%$ yield by addition of water and 5 equivalents of TFA after a reaction time of $30 \mathrm{~min}$. Overall, the alkynyltriazene can be seen in this process as a convenient and stable ketene synthon. Notably, a single diastereomer is formed, as confirmed by X-ray crystallographic analysis. Cyclobutanones are versatile intermediates, ${ }^{[13]}$ and as such, 14 can be further functionalized. Exposure of 14 to meta-chloroperoxybenzoic acid (mCPBA) delivered Baeyer-Villiger lactone $\mathbf{2 0}$ as a single regioisomer. The addition of catalytic amounts of $\left[\mathrm{Rh}(\mathrm{COD}) \mathrm{Cl}_{2}\right.$ in combination with 1,3-bis(diphenylphosphino)propane (dppp) and diphenyl acetylene promote regioselective activation of the proximal acylcarbon bond. Subsequent strain-releasing $\beta$-alkoxy elimination and reductive elimination formed lactone 21 in $94 \%$ yield.

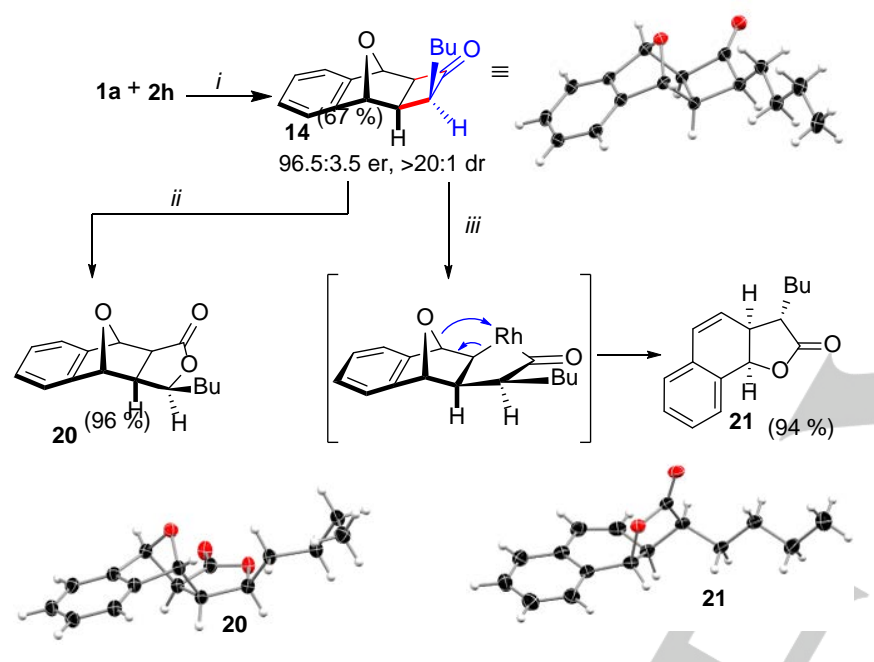

Scheme 5. One-pot cyclobutanone synthesis and product derivatisation: $i) \mathbf{2 h}$ $(0.1 \mathrm{mmol}), 1 \mathbf{a}(0.12 \mathrm{mmol}), 5 \mathrm{~mol} \% \mathrm{Ru}, \mathrm{Bu}_{4} \mathrm{NCl}(8 \mathrm{~mol} \%)$, THF $(0.3 \mathrm{M}), 23{ }^{\circ} \mathrm{C}$ $30 \mathrm{~min}$, then $\mathrm{H}_{2} \mathrm{O}$, TFA $(0.5 \mathrm{mmol}), 10 \mathrm{~min}, 23^{\circ} \mathrm{C}$; ii) $14(25.0 \mu \mathrm{mol}), \mathrm{mCPBA}$ $(50 \mu \mathrm{mol}), \mathrm{NaHCO}_{3}, \mathrm{DCM}, 0^{\circ} \mathrm{C}, 2 \mathrm{~h}$; iii) $14(50.0 \mu \mathrm{mol}), 5 \mathrm{~mol} \%[\mathrm{Rh}(\mathrm{COD}) \mathrm{Cl}]_{2}$, $12 \mathrm{~mol} \%$ dppp, $20 \mathrm{~mol} \%$ diphenylacetylene, dioxane, $120^{\circ} \mathrm{C}, 15 \mathrm{~h}$.

In summary, we have shown that 1-alkynyltriazenes are wellsuited substrates for enantioselective CpRu"-catalyzed [2+2] cycloaddition reactions with bicyclic alkenes. High yields and enantioselectivities were obtained, giving access to a broad range of cyclobutenyl triazenes. A salient feature of vinyl triazenes is the possibility to use them as vinyl cation surrogates. Under acidic conditions, they react cleanly with a broad range of nucleophiles, allowing the elaboration with different functional groups of high synthetic utility. This work lays a foundation for the use of vinyl triazenes as versatile synthetic intermediates.

\section{Acknowledgements}

This work was supported by the Swiss National Science Foundation ( $\mathrm{N}^{\circ} 157741$ and 175477) and the Ecole Polytechnique Fédérale de Lausanne (EPFL). We thank Dr. F.-T.
Farzaneh for help with the X-ray crystallographic analyses of compounds 3ca, 14, 20, 21.

Keywords: asymmetric catalysis $\bullet$ cycloaddition reactions • divergent synthesis $\bullet$ triazenes $\bullet$ ruthenium

[1] For reviews see: a) D. K. Kölmel, N. Jung, S. Bräse, Austr. J. Chem. 2014, 67, 328-336; b) S. Bräse, Acc. Chem. Res. 2004, 37, 805-816; c) D. B. Kimball, M. M. Haley, Angew. Chem. Int. Ed. 2002, 41, 33383351; Angew. Chem. 2002, 114, 3484-3498.

[2] For selected examples see: a) D. Wang, S. Cui, Tetrahedron 2016, 72, 2725-2730; b) H. T. Dao, P. S. Baran, Angew. Chem. Int. Ed. 2014, 53 14382-14386; Angew. Chem. 2014, 126, 14610-14614; c) C. Wang, H. Chen, Z. Wang, J. Chen, Y. Huang, Angew. Chem. Int. Ed. 2012, 51, 7242-7245; Angew. Chem. 2012, 124, 7354-7357 d) C.-Y. Liu, P. Knochel, J. Org. Chem. 2007, 72, 7106-7115; e) S. Bräse, S. Dahmen, J. Heuts, Tetrahedron Lett. 1999, 40, 6201-6203.

[3] K. Bott, Angew. Chem. Int. Ed. Engl. 1979, 18, 259-265; Angew. Chem. 1979, 91, 279-285.

[4] a) C. C. Lee, C. A. Obafemi, Can. J. Chem. 1981, 59, 1636-1640; b) C. C. Lee, E. C. F. Ko, Can. J. Chem. 1976, 54, 3041-3044; c) C. C. Lee A. J. Cessna, B. A. Davis, M. Oka, Can. J. Chem. 1974, 52, 2679 2683; d) W. M. Jones, D. D. Maness, J. Am. Chem. Soc. 1970, 92, 5457-5464; e) W. M. Jones, F. W. Miller, J. Am. Chem. Soc. 1967, 89, 1960-1962.

[5] G. Kiefer, T. Riedel, P. J. Dyson, R. Scopelliti, K. Severin, Angew. Chem. Int. Ed. 2015, 54, 302-305; Angew. Chem. 2015, 127, 306-310.

[6] F. Perrin, G. Kiefer, L. Jeanbourquin, S. Racine, D. Perrotta, J. Waser, R. Scopelliti, K. Severin, Angew. Chem. Int. Ed. 2015, 54, 13393-13396; Angew. Chem. 2015, 127, 13591-13594.

[7] a) D. Kossler, N. Cramer, Chem. Sci. 2017, 8, 1862-1866; b) D Kossler, N. Cramer, J. Am. Chem. Soc. 2015, 137, 12478-12481.

[8] a) C. G. Newton, D. Kossler, N. Cramer, J. Am. Chem. Soc. 2016, 138, 3935-3941; b) B. Ye, N. Cramer, Acc. Chem. Res. 2015, 48, 1308 1318; c) B. Ye, N. Cramer, J. Am. Chem. Soc. 2013, 135, 636-639; d) B. Ye, N. Cramer, Science 2012, 338, 504-506.

[9] For reviews see: a) K. Parthasarathy, C.-H. Cheng, Compr. Org. Syn. II 2014, 5, 222-272; b) W. Tam, J. Goodreid, N. Cockburn, Curr. Org Synth. 2009, 6, 219-238; c) P. Hu, Y.-H. Long, H. Wang, H.-H. Mo, D. Q. Yang, Chin. J. Org. Chem. 2008, 28, 1181-1192; d) T.-a. Mitsudo, Y. Ura, T. Kondo, Proc. Jpn. Acad. B. 2007, 83, 65-76; e) T.-a. Mitsudo, Y. Hori, Y. Watanabe, J. Organomet. Chem. 1987, 334, 157-167.

[10] For selected recent examples see: a) J. Goodreid, K. Villeneuve, E. Carlson, W. Tam, J. Org .Chem. 2014, 79, 10002-10012; b) G. C. Tsui, K. Villeneuve, E. Carlson, W. Tam, Organometallics 2014, 33, 3847-3856; c) R. Durham, J. Mandel, N. Blanchard, W. Tam, Can. J. Chem. 2011, 89, 1494-1505; d) N. Cockburn, E. Karimi, W. Tam, J. Org. Chem. 2009, 74, 5762-5765; e) A. Allen, K. Villeneuve, N Cockburn, E. Fatila, N. Riddel, W. Tam, Eur. J. Org. Chem. 2008, 4178-4192; f) R. R. Burton, W. Tam, Org. Lett. 2007, 9, 3287-3290; f) K. Villeneuve, W. Tam, Angew. Chem. Int. Ed. 2004, 43, 610-613; Angew. Chem. 2004, 116, 620-623.

[11] CCDC 1527105, 1550379, 1550380, 1550381 contain the supplementary crystallographic data for this paper. These data can be obtained free of charge from The Cambridge Crystallographic Data Centre via www.ccdc.cam.ac.uk/data_request/cif.

[12] a) M. Hanak, E. J. Carnahan, A. Krowczynski, W. Schoberth, L. R. Subramanian, K. Subramanian, J. Am. Chem. Soc. 1979, 101, 100 108; b) P. J. Stang, Z. Rappoport, M. Hanack, L. R. Subramanian, Vinyl Cations, Academic Press, New York, 1979.

[13] a) M. H. Shaw, J. F. Bower, Chem. Commun. 2016, 52, 10817-10829; b) T. Seiser, T. Saget, D. N. Tran, N. Cramer, Angew. Chem. Int. Ed. 2011, 50, 7740-7752; Angew. Chem., 2011, 123, 7884-7896; c) J. C Namyslo, D. E. Kaufmann, Chem. Rev. 2003, 103, 1485-1537; d) E. Lee-Ruff, G. Mladenova, Chem. Rev. 2003, 103, 1449-1484. 


\section{Entry for the Table of Contents}

\section{COMMUNICATION}

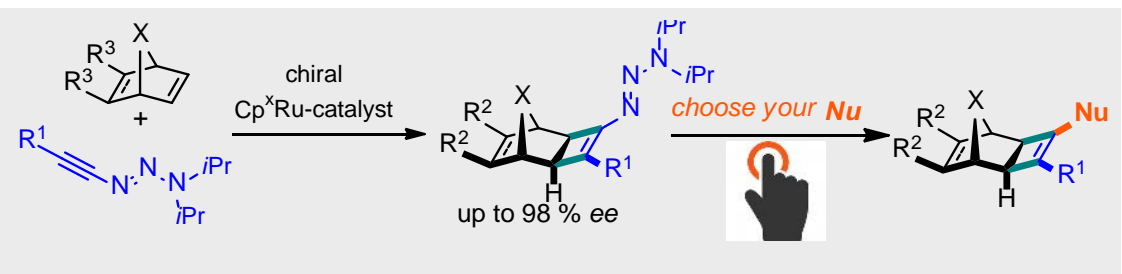

1-Alkynyltriazenes and bicyclic alkenes react in enantioselective [2+2] cycloadditions catalyzed by a chiral cyclopentadienyl Ru"-complex to give vinyl triazenes. The triazene functionality is readily replaced by a broad variety of nucleophiles including halides, alkoxides, sulfoxides, amides, arenes and heteroarenes, providing efficient access to a pool of chiral polycyclic compounds.
D. Kossler, F. G. Perrin, A. A.

Suleymanov, G. Kiefer, R. Scopelliti, K. Severin, * and N. Cramer*

Page No. - Page No.

Divergent Asymmetric Synthesis of Polycyclic Compounds via Vinyl Triazenes 